\title{
CAR mediates efficient tumor engraftment of mesenchymal type lung cancer cells
}

\author{
Mysore S Veena ${ }^{1}$, Min Qin², Åsa Andersson², Sherven Sharma ${ }^{2}$ and Raj K Batra ${ }^{2,3}$
}

The coxsackie-adenovirus receptor (CAR) is a developmentally regulated intercellular adhesion molecule that was previously observed to be required for efficient tumor formation. To confirm that observation, we compared the tumorigenicity of clonally derived test and control cell subsets that were genetically modified for CAR. Silencing CAR in lung cancer cells with high constitutive expression reduced engraftment efficiency. Conversely, overexpressing CAR in lung cancer cells with low constitutive expression did not affect tumor formation or growth kinetics. A blocking antibody to the extracellular domain of CAR inhibited tumor engraftment, implicating that domain as being important to this process. However, differences in adhesion properties attributable to this domain (barrier function and aggregation) could not be distinguished in the test groups in vitro, and the mechanisms underlying CAR's contribution to tumor engraftment remain elusive. Because high CAR cells displayed a spindle-shaped morphology at baseline, we considered whether this expression was an accompaniment of other mesenchymal features in these lung cancer cells. Molecular correlates of CAR were compared in model epithelial and mesenchymal type lung cancer cells. CAR expression is associated with an absence of E-cadherin, diminished expression of $\alpha$ - and $\gamma$-catenin, and increased Zeb1, Snail, and vimentin expression in lung cancer cells. In contrast, epithelial type (NCl-H292, Calu3) lung cancer cells show comparatively low CAR expression. These data suggest that if the mesenchymal cell phenotype is an accurate measure of an undifferentiated and invasive state, then CAR expression may be more closely aligned with this phenotype of lung cancer cells.

Laboratory Investigation (2009) 89, 875-886; doi:10.1038/labinvest.2009.56; published online 8 June 2009

KEYWORDS: lung cancer; tumorigenesis; EMT; CAR; adhesion molecules

CAR, the coxsackie-adenovirus receptor, is both a regulator of the Notch signaling pathway ${ }^{1,2}$ with roles in embryonic development, and an epithelial intercellular adhesion molecule with reported contributions to mucosal integrity and barrier function. ${ }^{3,4}$ It is a single-pass transmembrane protein initially described as the high affinity attachment receptor for coxsackie and group C adenoviruses (Ad). ${ }^{5}$ CAR is essential and embryonic stem cell-CAR knockouts are nonviable secondary to abnormal myocardial development. ${ }^{6-8}$

The epithelial-mesenchymal transition (EMT) is a phenotypic shift in which epithelial cells break down cell-cell and cell-extracellular matrix connections and then migrate to other locations in the body. These processes are known to be important for embryonic development, and are believed to contribute to repair of tissue injury and to invasive and metastatic tumor growth. EMT is modeled in epithelial cells by exposure to certain cytokines (eg, TGF $\beta 1$ ), or by exogenous expression of various transcription factors (eg, Twist or Snail), which can increase the invasive behavior of epithelial cells.

The dynamics of CAR expression in epithelial transitions is not clear. CAR is believed to recruit the tight junction (TJ) protein ZO-1 to the apical junctional complex. ${ }^{3}$ Imaging analyses localize CAR to the TJ in T-84 colon cancer cells, ${ }^{3}$ or to the adherens junctions (AJ) in A549 lung cancer cells. ${ }^{4}$ Although structurally distinct, ${ }^{9}$ functional analogies of CAR to E-cadherin or E-selectin have been suggested. ${ }^{4,10}$ CAR overexpression in $\mathrm{CHO}$ cells resulted in cellular aggregation; $^{3}$ overexpression in MDCK cells evidenced increased transepithelial electric resistance (TEER). ${ }^{3}$

\footnotetext{
${ }^{1}$ Department of Surgery, Geffen School of Medicine, University of California at Los Angeles, Los Angeles, CA, USA; ${ }^{2}$ Division of Pulmonary and Critical Care Medicine, Department of Medicine, Research/Development, Veterans Administration Greater Los Angeles Healthcare System, Los Angeles, CA, USA and ${ }^{3}$ Jonsson Comprehensive Cancer Center, Los Angeles, CA, USA

Correspondence: Dr RK Batra, MD, Geffen School of Medicine at UCLA and Division of Pulmonary and Critical Care Medicine, Veterans Administration Greater Los Angeles Health Care System, 11301 Wilshire Blvd 111Q, Los Angeles, CA 90073, USA.
}

E-mail: rbatra@ucla.edu

Received 13 January 2009; revised 11 March 2009; accepted 09 April 2009 
At intercellular junctions, the extracellular CAR N-terminal domains are believed to homodimerize to mediate homotypic cell adhesion and barrier function. The CAR intracellular C terminus contains PDZ-binding motifs for the initiation of structural and/or signaling scaffolds, ${ }^{11}$ but to date, signaling cascades emanating from CAR interactions have not been reported. Screens for CAR molecular interactions have been inconsistent; putative binding partners vary based on the assay system (deletion analyses, yeast two-hybrid screens, affinity chromatography or immunoprecipitation assays) as well as the cell model system that have been used. ${ }^{1-4,11-15}$ Proteins identified as putative CARbinding partners include several PDZ domain-containing proteins (ZO-1, MUPP-1, Ligand-of-Numb protein X, MAGI-1b, and PICK-1), and the functional implications of these interactions are currently under investigation.

CAR's contribution to the malignant phenotype is also under study. CAR is variably expressed in diverse tumors of both epithelial and mesenchymal origins. ${ }^{16-27}$ However, CAR intracellular localization, partners in protein interactions, physiological function in epithelia, or aberrant role in epithelial tumors remain largely unexplored. There have been several reports that have sought to link or correlate CAR expression with differentiation, tumorigenicity, and/or tumor progression. For example, based on cell culture models, a group of investigators has postulated that CAR is a global tumor suppressor in prostate and bladder cancer. ${ }^{16-18}$ Yet another group reports upregulated CAR expression with both tumor progression ${ }^{28}$ and with the inhibition of $\mathrm{EMT}^{29}$ in model systems. Another group evidenced enhanced CAR expression in the transition from preneoplastic lesions to invasive adenocarcinoma in a breast cancer model. ${ }^{30}$ These observations have led some to propose that CAR is a marker of tumor differentiation. ${ }^{31,32}$ However, we speculate that collectively, these paradoxical observations are reflective of a complex temporally limited expression of CAR in epithelia and in epithelial transitions.

We previously determined that (1) a soluble CAR ectodomain could be isolated from the tumor milieu (primary malignant pleural effusions), ${ }^{33}$ and (2) silencing CAR in human lung cancer cells impaired subcutaneous tumorigenesis in a scid mouse model. ${ }^{34}$ This impairment in tumor formation was not associated with significant differences in proliferation or induced apoptosis (by serum starvation) between control and test (CAR-silenced) cells. To confirm and expand upon our initial observation, we genetically modified selected lung cancer cells to derive clonal test and control populations that differed in CAR expression. After validation, these groups were tested in tumorigenicity and aggregation assays. Here, we report that silencing CAR in lung cancer cells with high CAR expression (NCI-H1703 and NCI-H157 cells) significantly impairs their ability to form tumors in vivo; whereas overexpressing CAR in cells with low constitutive CAR expression (NCI-H2122) does not have a measurable effect on tumorigenicity. Similarly, blocking antibody to the CAR ectodomain inhibits tumor formation by cells with high CAR, but not cells with low CAR. The spindle-shaped morphology of cells that showed high CAR contrasted with epitheliod features of cells that showed low CAR prompted us to consider whether CAR is associated with EMT in lung cancer cells. We compared the NCI-H1703 and NCI-H157 (high CAR) cells with validated lung epitheliod models (NCI-H292 and Calu3) for correlating CAR with the morphological and molecular features of EMT. Our data suggest that high CAR expression is closely associated with mesenchymal markers.

\section{MATERIALS AND METHODS Cells}

NCI-H1703, NCI-H157, NCI-H2122 non-small-cell lung cancer (NSCLC) cells (gifted by Dr Herbert Oie, NCI), and NCIH292 and Calu3 NSCLC cells (gifted by Dr K Krysan, Dubinett lab, UCLA Lung Cancer Research Program) were maintained in RPMI 1640 (Irvine Scientific, Santa Ana, CA, USA) with 10\% fetal bovine serum (FBS; Gemini, Woodland CA, USA) and penicillin $(100 \mathrm{U} / \mathrm{ml}) /$ streptomycin $\quad(100 \mu \mathrm{g} / \mathrm{ml}) \quad$ (complete medium). Morphological and phenotypic features relevant to the NCI-H1703, NCI-H157, and NCI-H2122 cells are described in the 'Results' section. The epitheliod cell models (NCI-H292 and Calu3 NSCLC cells) were chosen because they are reported to form functional junctions, and both lines have been extensively used as models of pulmonary epithelia. H292 cells are polarized in culture, ${ }^{35}$ and express E-cadherin with functional $\mathrm{AJ}^{36-38}$ Calu3 cells express functional TJs (with circumferential ZO-1 expression, high TEER, and low paracellular permeability characteristics), ${ }^{39}$ along with zonulae adherens, and desmosomes. ${ }^{36,40}$ Accordingly, Calu3 cells are a model of a tight lung epithelium.

\section{Vectors and Clonal Selection}

For CAR gene modification, we used an amphotropic retroviral vector encoding the full-length CAR open reading frame in forward and reverse orientations. Briefly, the fulllength CAR cDNA insert was digested (BamH1, Xho1) out of pcDNA3-CAR (gifted by Joanne Douglas, UAB Gene Therapy Center, Birmingham, AL, USA), and ligated into a linearized pLEGFP-N1 fragment in forward or reverse orientations downstream of the CMV promoter/enhancer sequence (Clontech, Becton Dickenson Biosciences, Palo Alto, CA, USA). Plasmid DNA was isolated from transformed DH $5 \alpha$, and insert orientations were confirmed with $\mathrm{Xba} 1$ digestion. Vectors were amplified and transfected (Effectene reagent; Qiagen, Valencia, CA, USA) into the PT-67 retroviral packaging cell line (Clontech, Becton Dickenson Biosciences). Producer cell supernatants were used to stably transduce lung cancer cells, and pooled populations were selected following G418 selection ( $500 \mu \mathrm{g} / \mathrm{ml})$ over 4 weeks. Clones were derived by limited dilution ( 1 cell per $\mathrm{ml}$ seeded as $200 \mu \mathrm{l}$ aliquots in 96 -well plates). Of these, 15-40 distinct colonies were characterized for CAR by immunoblot and/or 
flow cytometry (see below), and selected clonal populations were expanded for these studies.

\section{Characterization of CAR Gene-Modified Cells}

Transduction was confirmed by the presence or absence of $C A R$ gene amplification by DNA-polymerase chain reaction (PCR) primed by sequences flanking the multiple cloning sites in the pLEGFP-N1 vector. CAR expression was also functionally validated by Ad transduction in some cells; Ad vectors (Ad5LacZ) were amplified in the UCLA-Jonsson Comprehensive Cancer Center and stored at $-20^{\circ} \mathrm{C}$ in a nonfreezing solution containing $25 \%$ glycerol, $0.05 \%$ BSA, $4 \mathrm{M} \mathrm{CsCl}, 50 \mathrm{mM} \mathrm{NaCl}, 0.5 \mathrm{mM} \mathrm{MgCl}_{2}$, and $5 \mathrm{mM}$ Tris buffer as described. ${ }^{19}$ Vectors were gel-filtered (G-50 Sephadex; Boehringer Mannheim, Indianapolis, IN, USA) and eluted into growth medium for transduction of target cells at a multiplicity of infectious units of 100. All gene-modified clones were validated for CAR by PCR, immunoblot of cell lysates, and/or by surface CAR expression by flow cytometry. For RT-PCR, total RNA was extracted from control or CARsilenced clones using TRIzol reagent (Life Technologies, Grand Island, NY, USA). RT was performed using the Super Script One Step RT-PCR system (Gibco-BRL, Grand Island, NY, USA). Primers for CAR expression were as follows: sense: $5^{\prime}$-ATGCCCACTTCATGGTTAGC- ${ }^{\prime}$; antisense: $5^{\prime}$-TAGGG GCAGCTACCTTAGCA- $3^{\prime}$. For GAPDH control, sense: $5^{\prime}$-C CACCCATGGCAAATTCCATGGCA-3' ${ }^{\prime}$, antisense: $5^{\prime}$-TCTAG ACGGCAGGTCAGGTCCACC- $3^{\prime}$ were used. The RT-PCR protocol was as follows: cDNA synthesis (one cycle: $54^{\circ} \mathrm{C}$ for $30 \mathrm{~min}$ ) followed by $94^{\circ} \mathrm{C}$ for $2 \mathrm{~min}$. DNA amplification followed $\left(25\right.$ cycles: $94^{\circ} \mathrm{C}$ for $15 \mathrm{~s}$, anneal $55^{\circ} \mathrm{C}$ for $30 \mathrm{~s}$, extend $72^{\circ} \mathrm{C}$ for $1 \mathrm{~min}$ ) with a final extension at $72^{\circ} \mathrm{C}$ for $8 \mathrm{~min}$.

For flow cytometry, surface CAR was labeled ${ }^{34}$ using $\mathrm{RmcB},{ }^{41}$ a murine monoclonal antibody that recognizes the extracellular N-terminal region of CAR. Briefly, $1 \times 10^{6}$ cells were preincubated in $100 \mu \mathrm{l} 0.1 \% \mathrm{BSA}$ (in PBS for $20 \mathrm{~min}$ at room temperature) before primary antibody $(\mathrm{RmcB}$; mouse IgG1, $1: 100$ dilution in $\mathrm{PBS} / 0.1 \% \mathrm{BSA}$ for $90 \mathrm{~min}$ at room temperature) was admixed on an orbital shaker. Cells were then sedimented and washed three times with $\mathrm{PBS} / 0.1 \%$ $\mathrm{BSA}$, incubated with secondary (PE-conjugated sheep antimouse $\left(\mathrm{Fab}^{\prime}\right)_{2}$; Sigma, St Louis, MI, USA, 1:200 dilution in $\mathrm{PBS} / 0.1 \%$ BSA for $30 \mathrm{~min}$ at room temperature in the dark), washed three times and resuspended in $500 \mu \mathrm{l}$ PBS for flow cytometry by FACScan using CellQuest software (Becton Dickinson, Mountain View, CA, USA). For all data acquisition, gates were based on the forward and side scatter profiles of unstained cells, and surface expression of target proteins was normalized to cells that had been incubated with secondary antibody alone.

\section{Antibody-Mediated CAR Neutralization and Tumorigenesis Assays}

For comparative tumorigenesis, 6-week-old $n u / n u$ or scid/ scid mice were injected with $5 \times 10^{6}$ to $8 \times 10^{6}$ cells per mouse subcutaneously, in the flank region, in groups of 4-6, under an institutionally approved protocol. The predefined primary outcome measurement was tumor volumes at the time point when the first mouse in either the control or test groups met institutional criteria for killing due to tumor burden. Tumor dimensions were estimated based on bisecting diameters measured with a caliper, and the tumor volume was approximated using the formula $0.4\left(a b^{2}\right)$ where $a$ is the long measured axis of the tumor and $b$ the short measured axis. For CAR-blocking antibody $(\mathrm{RmcB})$ blockade experiments, $5 \times 10^{6}$ NSCLC cells were co-injected into the right flank subcutaneous region of female $n u / n u$ mice (four or five mice per group) with $2 \mu \mathrm{g}$ of RmcB or control murine IgG1. Tumor volumes were assessed every $24-48 \mathrm{~h}$ until mice in either test or control cohort met criteria for killing, or the experiment was terminated after a maximum of 90 days.

\section{Cell Aggregation Assays}

Cell aggregation assays were performed with minor modifications of methods described earlier. ${ }^{42,43}$ Briefly, cells grown to $85 \%$ confluence in complete medium were washed with $10 \mathrm{mM}$ HEPES buffer ( $\mathrm{pH}$ 7.4) containing $150 \mathrm{mM}$ $\mathrm{NaCl}, 5 \mathrm{mM} \mathrm{CaCl}_{2}$, and $1 \mathrm{mM} \mathrm{MgCl} 2$ (aggregation buffer), and then detached with $0.01 \%$ trypsin (Invitrogen, Carlsbad, CA, USA) in the same buffer on a gyratory shaker at 75 r.p.m. At the end of the incubation period, trypsin was quenched with $20 \% \mathrm{FBS} / \mathrm{RPMI}$, and cells were sedimented ( $200 \mathrm{~g}$ for $5 \mathrm{~min}$ ). The cell pellet was resuspended in the aggregation buffer containing $1 \%$ BSA; cell suspensions (density of $5 \times 10^{4}$ cells per $0.5 \mathrm{ml}$ ) were added to individual wells of a 24 -well plate and incubated at $37^{\circ} \mathrm{C}$ for $1 \mathrm{~h}$ on a gyratory shaker at 75 r.p.m. Cells were observed for aggregation in a Leica DMIL microscope equipped with Hamamatsu ORCA-ER digital camera. Photomicrographs were captured at $\times 40$ magnification using MetaMorph software. E-cadherin and CAR neutralization was performed based on published methods. ${ }^{19,33,34,41,44}$ Briefly, cells were incubated with blocking antibodies $(10 \mu \mathrm{g} / \mathrm{ml}$ of anti E-cadherin; Zymed Laboratories or $5 \mu \mathrm{g} / \mathrm{ml} \mathrm{RmcB}$ ) for $30 \mathrm{~min}$ before starting the aggregation. Cell aggregation was quantified by the measured decrease in the free or individual particle number (individual particles defined as aggregates $\leq 3$ cells) in high-powered microscopic fields. For these analyses, $10 \mu \mathrm{l}$ of a $5 \times 10^{4}$ cells per $0.5 \mathrm{ml}$ cell suspension (therefore, containing 1000 cells) was resuspended in $50 \mu$ l volume of aggregation buffer, and the total number of free/individual particles was counted. The percent aggregation was calculated as $1-(($ number of free/individual particles $) /($ initial particle number or 1000$)) \times 100)$.

\section{Molecular Characterization of EMT Markers Using Immunoblots}

Coordinated cell cultures were lysed following the end of $\log$ phase growth (post-confluent state, $24 \mathrm{~h}$ after culture confluence) using Mammalian Cell-PE LB medium 
(Genotechnology Inc., St Louis, MO, USA) at $4^{\circ} \mathrm{C}$ containing Complete Protease Inhibitor Cocktail (Roche Applied Science, Indianapolis, IN, USA). Insoluble debris was pelleted by centrifugation and total protein in the supernatant was quantified using the Bio-Rad Assay Kit (Bio-Rad, Hercules, CA, USA). Protein $(40 \mu \mathrm{g})$ was suspended in 1 volume of Laemmli sample buffer (Bio-Rad), and the volume of the samples was brought to $50 \mu \mathrm{l}$ with Mammalian Cell-PE LB medium for loading. The samples were heated at $95^{\circ} \mathrm{C}$ for 5 min, resolved by $10 \%$ SDS-PAGE, and transferred onto polyvinylidene difluoride membrane (Millipore Corp., Billerica, MA, USA) on ice. After blocking the membrane with NAP-Sure Blocker (Genotechnology Inc.), the membrane was washed three times in PBS and $0.1 \%$ Tween 20 (PBST) over $30 \mathrm{~min}$, and probed using the following primary antibodies (diluted in PBST as recommended by the manufacturer, overnight incubations at $4^{\circ} \mathrm{C}$ ): polyclonal anti-CAR $\mathrm{H} 300$, anti-Zeb H-102, anti-Occludin $\mathrm{H}-279$, anti- $\beta$-tubulin $\mathrm{H} 202$, and anti-vimentin V7 were from Santa Cruz Biotechnology (Santa Cruz, CA, USA), anti-E-cadherin (BD Biosciences PharMingen/Transduction Laboratories, San Jose, CA, USA), anti- $\beta$-catenin and anti-snail (Cell Signaling, Danvers, MA, USA), anti- $\alpha$-catenin (Chemicon International, Temecula, CA, USA), anti- $\gamma$-catenin and anti-ZO-1 (Zymed/Invitrogen Laboratories, Carlsbad, CA, USA). Following three washes in PBST, the primary antibody was labeled with the appropriate horseradish-peroxidase-conjugated secondary antibody (1:5000 dilution, $1 \mathrm{~h}$ at room temperature; Amersham Biosciences, Piscataway, NJ, USA). After repeated washes in PBST, specific bands were detected by chemiluminescence (Amersham Biosciences).

\section{Statistical Methods}

The mean and standard deviation of categorical values (percent transduction, tumor volumes at time of first killing) were compared for differences using the unpaired $t$-test or one-way ANOVA, using GraphPad InStat software. A statistically significant difference was defined as $P<0.05$ between the groups compared.

\section{RESULTS}

\section{Silencing CAR in Lung Cancer Cells with High Expression} Impairs their Ability to Form Tumors

NCI-H1703 and NCI-H157 lung cancer cells (selected as models because they highly express CAR constitutively) were genetically silenced for CAR, and clones were isolated ( 1 cell per $\mathrm{ml}$ seeded as $200 \mu \mathrm{l}$ aliquots in 96-well plates). Colonies were expanded and clonally derived cell populations were validated for altered CAR expression. For the NCI-H1703 subset, we used Ad transduction as a functional screen for CAR expression, because CAR is the cellular attachment receptor for $\mathrm{Ad},{ }^{5}$ and because CAR expression is a primary determinant of Ad transduction efficiency of lung cancer cells in vitro and in vivo. ${ }^{20,45}$ CAR-silenced clones exhibited significant reductions in Ad transduction efficiency (Figure 1a) when compared with parental or vector controls. The decrease in Ad transduction efficiency was corroborated by marked reductions in CAR expression as directly measured by CAR-mRNA transcript by semiquantitative RT-PCR (Figure 1b), and by validation of CAR expression by flow cytometry (Figure 1c). Surface expression of CAR in the parental and vector control groups was confirmed to be significantly higher than the CAR-silenced cells, and significantly lower than the CAR-overexpressing cells.

Clonally derived populations of both CAR-silenced lung cancer cells (NCI-H1703, NCI-H157) were significantly inhibited in their ability to form subcutaneous xenografts, as compared to control populations (Figures 1d and e). For example, in the NCI-H1703 cohort, CAR silencing was associated with a reduction in the efficiency of tumor formation with two distinct clones (1/4 with H1703-AS5 and 1/5 tumors with H1703 AS6 cells), compared to parental and vector control (4/4 and 5/5, respectively). In addition, the aggregate tumor volume (Figure 1d) in the CAR-silenced groups was significantly reduced (at 40 days, when the control subset first met criteria for killing because of tumor burden). Silencing CAR in NCI-H157 cells also decreased engraftment efficiency (1/5 with H157 CAR-silenced clone, vs 4/4 with H157 vector control) and the aggregate tumor volume ( $P=0.008$ by the unpaired $t$-test), compared to vector control (Figure 1e). These results indicate that silencing CAR in lung cancer cells that display high constitutive expression impairs the engraftment efficiency of those cells. Tumors that arose from NCI-H157 overexpressing CAR $(\mathrm{CAR}+)$ followed a kinetic trend similar to that of the vector control cells (Figure 1e).

Interestingly, we observed the slow emergence of two tumors in mice that had been injected with the CAR-silenced (AS5 and AS6) cohorts, and the tumor cells from these late tumors showed high CAR expression (Figure 1f). Thus, when the delayed-onset tumors were extirpated (at day 101 or 118 after implantation), the surface CAR expression in the rescued cancer cells was similar to that of wild-type control, rather than that of the cells that were originally implanted (Figure 1f). These results seem to suggest that although CAR-silenced cells were initially inhibited in their ability to form tumors in vivo, the delayed tumors arose from phenotypic revertants that had possibly inactivated the CAR-silencing vector, and thus displayed surface CAR expression again.

\section{CAR Overexpression in NCI-H2122 Lung Cancer Cells does not Effect Subcutaneous Tumor Formation}

Similarly, CAR gene-modified clones were generated from lung cancer cells with very low constitutive CAR expression (H2122 cells); ${ }^{20,45}$ representative flow scattergrams of the grouped clonal cell populations are presented (Figure 2a). Silencing CAR in H2122 cells resulted in a cell population 

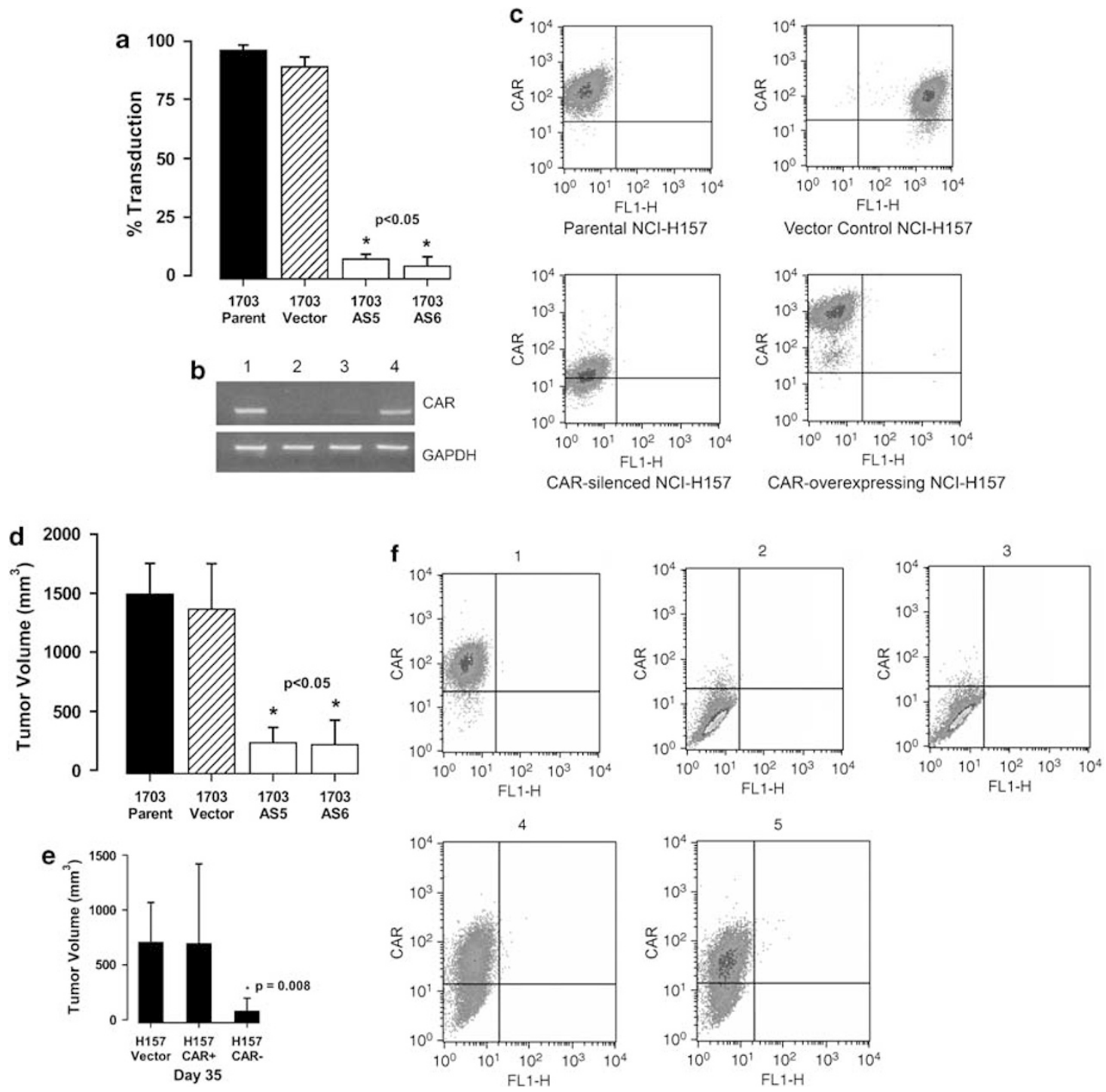

Figure 1 Silencing CAR in NCl-H1703 and NCl-H157 cells impairs their ability to form subcutaneous tumors: clonal test (CAR-silenced, CAR-overexpressing) or control (NCl-H1703, NCl-H157, or vector control) populations are validated for CAR expression by Ad transduction, RT-PCR, and/or flow cytometry. Following, the ability of these cell cohorts to generate subcutaneous xenografts in scid mice is evaluated. (a) Compared to parental and vector controls, CAR-silenced clones (1703 AS5 and 1703 AS6) are significantly more refractory to Ad transduction. (b) Compared to parental (lane 1) and vector (lane 4) controls, CAR-silenced clones display reduced levels of the CAR transcript. (c) For flow cytometry, $1 \times 10^{6}$ cells are labeled with primary antibody (RmcB), sedimented and washed, and detected with PE-conjugated sheep anti-mouse (Fab') ${ }_{2}$. Surface expression of CAR was normalized (at $>99$ th percentile) to cells that had been incubated with secondary antibody alone. Depicted is comparative CAR expression in the NCI-H157 (parental, vector control, CAR-silenced, and CAR-overexpressing) clones. CAR expression is on the ordinate scale; the green channel fluorescence (abscissa) in the vector control cells is attributable to the expression of the enhanced green fluorescent protein (EGFP) in control construct. (d) CAR-silenced NCl-H1703 clones (AS5 and AS6) are inhibited in their ability to engraft in scid mice. Depicted are the tumor volumes $\left(\mathrm{mm}^{3}\right) \pm$ standard deviation at day 40 after implantation. ${ }^{*} P<0.05$ for a significant difference between tumor formation by the vector control vs CAR-silenced cells. (e) CAR-silenced NCI-H157 clone (CAR-) are inhibited in their ability to engraft in scid mice. There is no significant difference in tumor growth between the control and CAR-overexpressing $(C A R+)$ clonal cohort. ${ }^{*} P=0.008$ for a significant difference between the vector control vs CAR-silenced cells at day 35 after implantation. (f) Delayed-onset tumors mice that were engrafted with CAR-silenced cells have cells that display high CAR. (f1), Wild-type (H1703 cells) CAR expression (mean channel fluorescence intensity or MCF $=108)$ in; $(f 2), C A R-s i l e n c e d ~ H 1703$ AS5 clone $(M C F=5)$ at time of implantation; ( $f 3)$, CAR-silenced H1703 AS6 clone $(M C F=5)$ at time of implantation; ( $f 4), C A R$ in tumor cells extirpated from H1703 AS5 xenografts (integrated MCF = 45); and (f5), CAR in tumor cells extirpated from $\mathrm{H} 1703$ AS6 xenografts (integrated MCF $=41$ ). These results suggest that reemergence of CAR expression may be associated with delayed tumor formation in these mice.

that could not be differentiated from the parental or vector control groups on the basis of the surface phenotype. Comparatively, there was significantly higher surface expression of
CAR $(\mathrm{MCF}=404)$ in the $\mathrm{H} 2122$ CAR-overexpressing population. However, overexpressing CAR in these cells did not impact either engraftment efficiency $(100 \%$ in every 
group) or tumor volumes (Figure $2 \mathrm{~b}$ ) in relation to controls, and the tumor kinetic curves were virtually superimposable across the groups (data not shown). Thus, genetically silencing or overexpressing CAR in cells with low constitutive expression has no measurable impact on engraftment efficiency or tumor growth.
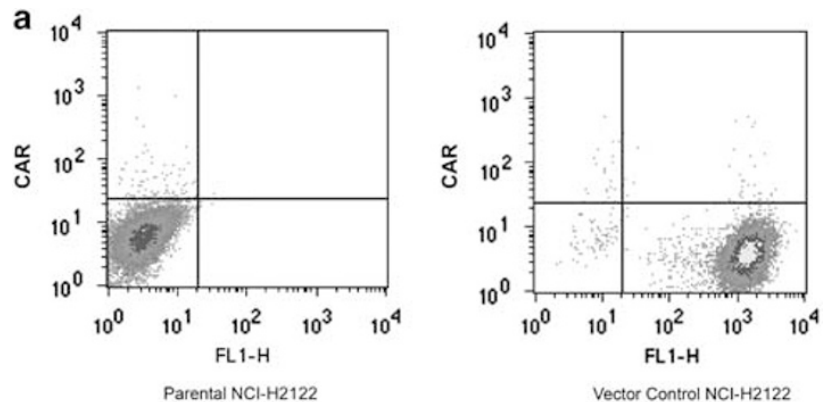

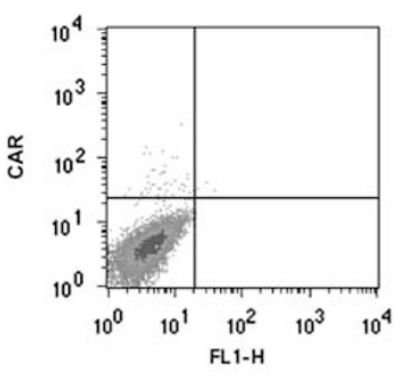

CAR-silenced NCl-H2122

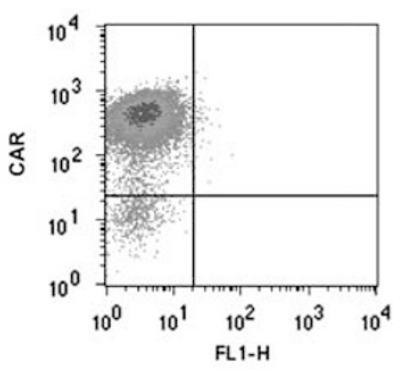

CAR-overexpressing NCl-H2122

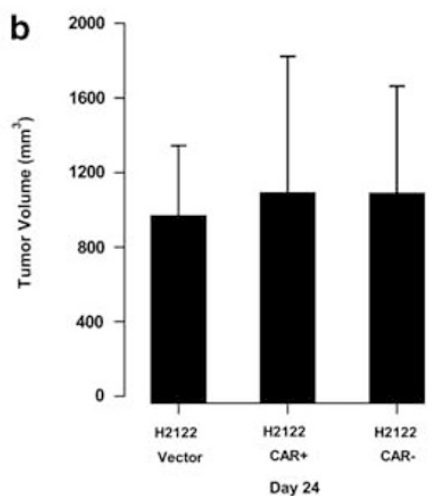

Figure 2 CAR overexpression in $\mathrm{NCl}-\mathrm{H} 2122$ lung cancer cells does not effect subcutaneous tumor formation. (a) Clone of CAR gene-modified $\mathrm{NCl}-\mathrm{H} 2122$ cells was propagated and validated for surface CAR expression. Depicted are representative flow scattergrams of $\mathrm{NCl}-\mathrm{H} 2122$ (parental, vector control, and CAR-silenced and CAR-overexpressing) clonally derived cell populations, with CAR expression represented in the ordinate scale. Parental group $(M C F=7)$, vector control $(M C F=4), C A R$-silenced $(M C F=4)$, and CAR-overexpressing (integrated MCF $=404$ ) cells. (b) Overexpressing or silencing CAR in lung cancer cells that have low CAR expression (NCI-H2122 cells) does not change their ability to form tumor xenografts. Vector control, CAR-overexpressing (H2122CAR +), and CAR-silenced (H2122CAR-) NCl-H2122 cells were injected into the right flank region of scid/scid mice. Tumor volumes were monitored every other day until the time when the first mouse in either the control or test groups met criteria for killing. Depicted are the mean tumor volume \pm standard deviation within each group of animals at day 24 after implantation.

\section{Blocking Antibody to CAR Extracellular Domain Impairs Tumor Formation in NCl-H1703 Cells, but not in NCl-H2122 Cells}

The distal extracellular domain of CAR on adjacent cells is envisioned to homodimerize and function in homotypic epithelial cell adhesion. ${ }^{46}$ This domain also mediates binding to coxsackievirus and subgroup $\mathrm{C}$ adenovirus with high avidity $(\mathrm{Kd} \sim 1 \mathrm{nM}),{ }^{5,41,47}$ a process which is blocked using the murine monoclonal antibody $\mathrm{RmcB} .{ }^{5,41}$ Thus, we used RmcB to block cell-cell interaction mediated by the distal extracellular domain of CAR. Compared to control murine IgG1, co-injection of RmcB with lung cancer cells unfailingly impaired tumorigenesis when those cells highly expressed CAR (Figures 3a,b). By contrast, RmcB neutralization of CAR-CAR interactions did not significantly alter the tumor kinetics of NCI-H2122 cells, which have low CAR expression (Figure 3c). The difference in the tumorigenicity was not attributable to differences in complement-mediated lysis of antibody-coated CAR + cells (H1703 and H157) vs CARdeficient $\mathrm{H} 2122$ cells. In this regard, there was no difference in rabbit HLA-ABC complement-mediated lysis with RmcB vs mIgG1 in vitro (data not shown). Notably, injecting RmcB into established tumors did not induce tumor regression, and growth of established tumors proceeded as with control mIgG1 (data not shown). Together, these results suggest that blocking of CAR extracellular domain function (previously reported by others as homotypic adhesion) may serve to block the initial engraftment of CAR-expressing tumors in vivo, but blocking CAR interactions when tumors have developed does not lead to tumor regression.

\section{CAR Adhesion Function cannot be Evidenced by In Vitro Measures of Barrier Function or Cell Aggregation in Lung Cancer Cells}

CAR overexpression in model epithelia has been shown to increase TEER, ${ }^{3}$ and heterologous expression in CHO cells is reported to bring about cell aggregation through homotypic cell adhesion. ${ }^{3,46}$ Because blocking the activity of the CAR extracellular domain reduced engraftment efficiency in vivo, we investigated whether we could develop a functional correlate of this activity in vitro, using lung cancer cell models. To control for the activity of E-cadherin in these functional analyses, we included the immunoblockade of E-cadherin into the assay design. CAR expression was not associated with the establishment of a transepithelial electrical resistance (TEER) in the lung cancer cell models (whereas coordinated cultures of MDCK cells generated peak resistances of $1361 \Omega \mathrm{cm}^{2}$ and plateau of $300-600 \Omega \mathrm{cm}^{2}$ for up to 2 weeks after the gradient had been established). In addition, we did not find CAR expression to be associated with increased cell aggregation in the lung cancer cells tested. Thus, the H1703 cells aggregated to a similar degree as the CAR-silenced cohorts derived from those cells (Figures $4 \mathrm{a}$ and $\mathrm{b}$ ), and there was no difference in cell aggregation between cohorts of NCI-H2122 cells and clones that were genetically modified to 
overexpress CAR (NCI-H2122S13) (Figures 4c and d). The suggestion that CAR was not significantly contributing to the in vitro aggregation function was also buttressed by blocking
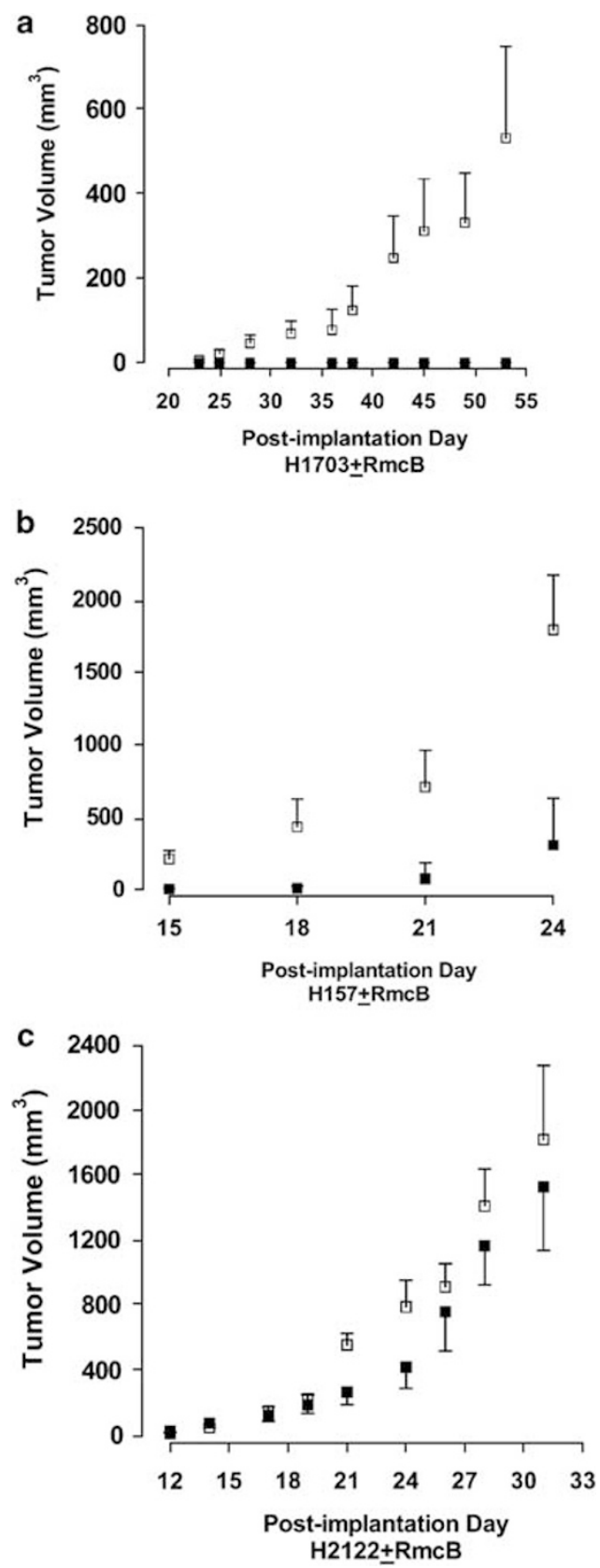

Figure 3 Blocking antibody to CAR extracellular domain impairs tumor formation by NSCLC cells with high constitutive CAR expression ( $\mathrm{NCl}$ $\mathrm{H} 1703, \mathrm{NCl}-\mathrm{H} 157)$, but not of cells with low CAR (NCl-H2122): RmcB, a murine monoclonal antibody that recognizes the CAR extracellular domain, was used to block CAR-CAR homotypic binding at the time of tumor implantation. A total of $5 \times 10^{6} \mathrm{NSCLC}$ cells $+2 \mu \mathrm{g}$ per $10^{6}$ cells of RmcB or control murine lgG1 were implanted into the right flanks of female $n u / n u$ mice. Implanted lung cancer cells (open squares) or cells + RmcB (solid squares) were monitored for tumor formation over time. Depicted are the tumor-growth curves (mean + standard deviation tumor volume) to a time point of first killing. (a) NCl-H1703 cell cohort (b) NCl-H157 cell cohort, (c) $\mathrm{NCl}-\mathrm{H} 2122$ cell cohort. experiments in the NCI-H2122 cell cohort. NCI-H2122 cells express E-cadherin; ${ }^{48}$ thus, we tested the extent to which the observed aggregation was attributable to E-cadherin vs CAR in H2122 cells overexpressing CAR (NCI-H2122S13) cells. Whereas the incubation of neutralizing antibody to E-cadherin largely blocked NCI-H2122S13 cell aggregation, blocking CAR did not have a significant effect on this function (Figures 4e and f). Despite differences in CAR expression, NCI-H1703 cells and their CAR-silenced derivatives aggregated (as defined by cell clusters $\geq 4$ cells) approximately $20 \%$ of the time, whereas both the NCI- 2122 cells and their CAR-overexpressing derivatives aggregated $80 \%$ of the time. These results indicate that changes in CAR expression cannot be measured by changes in paracellular ion flux (barrier electrical function) or in vitro cell aggregation in lung cancer cells. However, these data certainly do not exclude the possibility that CAR adhesion functions are important to tumor engraftment in vivo. a
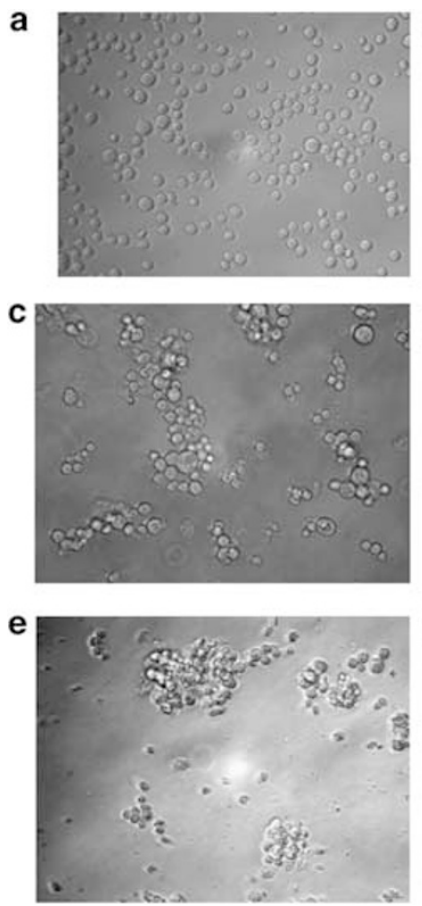


Figure 4 CAR expression is not associated with cell aggregation in lung cancer cells. Paired clones of CAR gene-modified lung cancer cells ( $\mathrm{NCl}-\mathrm{H} 1703$ and $\mathrm{NCl}-\mathrm{H} 1703$ AS6; NCl-H2122 and $\mathrm{NCl}-\mathrm{H} 2122 \mathrm{~S} 13$ ) were compared for cell aggregation in gyrating suspensions. The relative contribution of CAR or E-cadherin to cell aggregation in the $\mathrm{NCl}-\mathrm{H} 2122 \mathrm{~S} 13$ cells (which express both of these molecules) was assessed with blocking antibodies. Cell aggregation was quantified by the measured decrease in the free particle number in high-powered microscopic fields as described in the Materials and methods section. Depicted are photomicrographs following $1 \mathrm{~h}$ in a gyrating suspension. (a) NCl-H1703 cells, (b) NCl-H1703AS6 (CAR-silenced) cells, (c) NCl-H2122 cells, (d) NCl-H2122S13 (CAR-overexpressing) cells, (e) NCl-H2122S13 cells + RmcB (anti-CAR), (f) NCl-H2122S13 cells + anti-E-cadherin. CAR expression does not contribute to NSCLC cell aggregation in this in vitro assay. 
a Calu 3

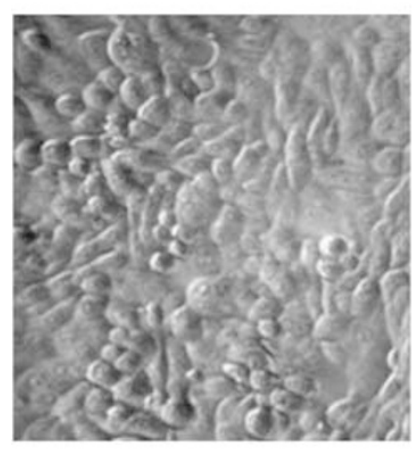

292

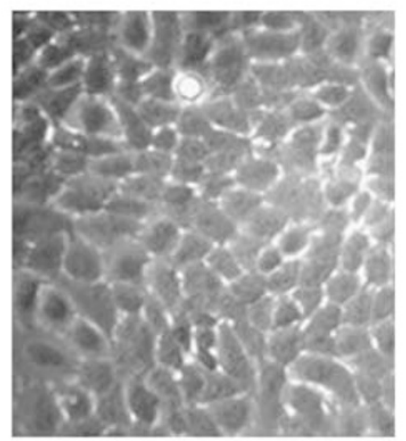

157

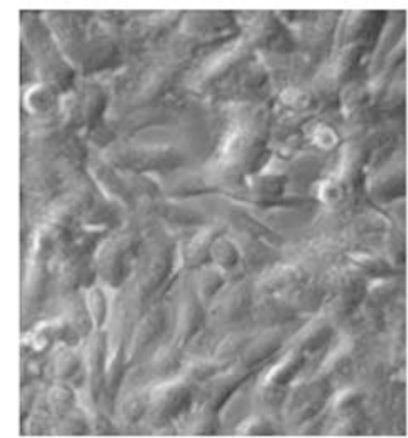

1703

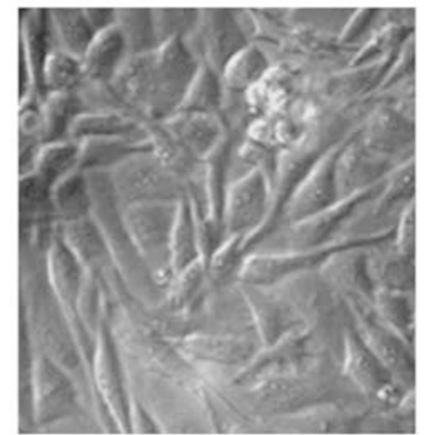

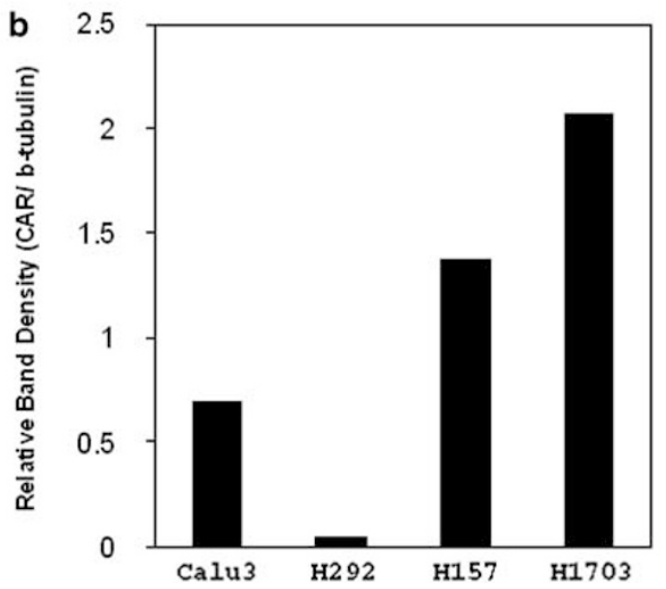

C

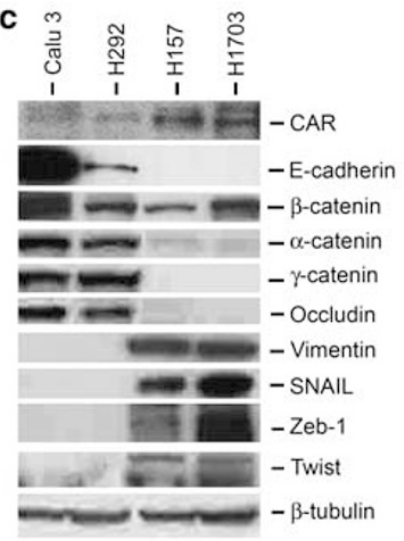

Figure 5 CAR expression is more closely associated with markers of the mesenchymal rather than the epitheliod phenotype of lung cancer cells. Lung cancer cells were grown to a post-confluence state, at which time cell lysates were extracted and quantified for total protein. Protein ( $40 \mu \mathrm{g})$ was separated on a $10 \%$ SDS-PAGE gel and probed for markers associated with mesenchymal or epitheliod phenotypes. (a) Photomicrographs of confluent cultures of $\mathrm{NCl}-\mathrm{H} 292$ and Calu3 (epitheliod morphology) vs NCI-H1703 and NCI-H157 (mesenchymal morphology) lung cancer cells. (b) Graphical depiction of differences in CAR expression, as indexed to control $\beta$-tubulin, between the lung cancer cell models. (c) Comparative immunoblots of epitheliod vs mesenchymal markers in the lung cancer cell models.

\section{CAR Expression Correlates with a Molecular Signature Suggesting a Mesenchymal Phenotype}

The function of CAR in conferring the malignant phenotype is unclear. Whereas increased CAR expression has been associated with the malignant conversion of breast epithelium, ${ }^{28,30}$ the prevailing view is that CAR is a marker of tumor differentiation. ${ }^{22,31,32}$ In lung cancer cells that display high constitutive expression, we have associated CAR with successful engraftment in vivo ${ }^{34}$ (Figures 1 and 3). Moreover, we were struck by the observation that lung cancer cells that exhibit high CAR are morphologically more akin to mesenchymal rather than epitheliod tumor cells, and the NCI-H2122 cells, which express low CAR, are more representative of epitheliod cells. ${ }^{49}$ This observation prompted us to consider the extent to which CAR is evident in lung cancer cells that have been functionally characterized as epithelial. Both NCI-H292 and Calu3 lung cancer cells have been extensively used as experimental models of pulmonary epithelia; these cells polarize in culture, and express functional tight and/or adherens junctions. ${ }^{35-40}$ All protein lysates in the lung cancer models were extracted when cultures had reached a post-confluence state (Figure 5a), to minimize the impact of density-dependent differences in marker expression. CAR expression was highest in mesenchymal type (fibroblastoid) cells (NCI-H1703 and NCI-H157 cells; Figure $5 \mathrm{a}, \mathrm{b}$ ), and was directly related to mesenchymal markers (vimentin and transcriptional repressors of E-cadherin) (Figure 5c). Conversely, the confluent epitheliod cell lines poorly expressed CAR by comparison. As a result, CAR expression inversely correlated with junctional (E-cadherin, occludin, and $\alpha$ - and $\gamma$-catenin) (Figure $5 \mathrm{a}$ and b) protein expression. Total $\beta$-catenin expression was not significantly different between the epitheliod or fibroblastoid cells. These data suggest that the intercellular adhesion molecule CAR is more highly expressed in the lung cancer subset that shows mesenchymal characteristics.

\section{DISCUSSION}

Malignant transformation and tumor progression is associated with acquired alterations in cell-cell interactions, which impact the organization and functionality of epithelium. Tumors differ from tissues of their epithelial origin by 
using proteins that function out of context with tissue development, that have perturbations in the expression and/or cellular localization, or that show abnormalities in physiological (protein-protein) interactions. These processes underlie tumor growth as well as progression to invasion and metastases.

CAR is an immunoglobulin-like single-spanning transmembrane intercellular adhesion molecule that has an essential function in development. CAR's role has primarily been investigated in the context of Ad gene delivery; however, its physiological and pathological functions (eg, in malignancy) remain unclear. Recently, several studies that examine the expression of CAR in human epithelial tumors have been published. For example, a group of investigators has compared CAR immunohistochemistry in prostate epithelium and prostate cancer in a grade-specific and metastases-associated manner. They evidence high CAR expression in prostate epithelium, diminishing CAR with increasing grade of primary prostate cancer, and the re-emergence of high CAR expression in metastases. ${ }^{22}$ Their interpretation of these results is that CAR expression may be analogous to E-cadherin with respect to epithelial function and cellular differentiation. ${ }^{22}$ Our data and speculative hypotheses are not consistent with this vision of CAR biology (see below). In another study, archived breast cancer tissues showed higher amounts of CAR transcripts when compared to background, and CAR expression trended with an increase tumor grade and metastases. ${ }^{50}$ Paradoxically, however, the report also suggested that levels of CAR positively correlated with survival. ${ }^{50}$ Similarly, in lung cancer, Wang et al ${ }^{51}$ suggest that CAR expression may be subtype associated, a finding that is consistent with an observation in cell culture models reported over a decade ago. ${ }^{19}$ Thus, whereas squamous-cell and small-cell lung cancers highly expressed CAR; only a minority of adenocarcinomas showed detectable expression. Intensity of CAR labeling did not appear to correlate with tumor grade, and importantly, CAR expression in inflammatory or hemorrhagic lung injury was not observed to be increased. ${ }^{51}$ However, in adenocarcinomas of a different tissue origin, a majority of endometrial tumors displayed CAR expression, with both a membranous and a cytoplasmic pattern of distribution. ${ }^{52}$ Accordingly, a consensus agreement regarding CAR's role in epithelial function and/or pathological contributions is difficult to glean from these diverse observations and interpretations.

Personal discussions with an investigator (Dr David Beer, University of Michigan) who has molecularly profiled resected lung cancer specimens, combined with our own unpublished preliminary data seem to suggest that CAR is variably expressed in lung cancer cells. Across a large panel of resected lung cancers (thus, representing a sampling bias toward well-differentiated and circumscribed lung cancers), CAR expression is highly variable, not unlike that of E-cadherin. In fact, CAR expression seems to be directly correlated with E-cadherin expression in resected adenocarcinomas, but not lung squamous-cell carcinomas. We speculate that differences in CAR expression are reflective of varying degrees of intratumoral heterogeneity among various tumors. If that speculative hypothesis is correct, then perhaps the most relevant question is not 'what subtype of tumors highly express CAR?', but rather 'what are the distinguishing features of CAR-expressing cells in an individual tumor?' We speculate that the CAR-expressing cell subset may have unique properties that impact clinical behavior (eg, tumor progression and/or metastases). Given our long-standing focus on lung cancer, in which intratumoral heterogeneity is the rule rather than the exception, we seek to examine the functional impact of differences in CAR expression in future studies.

Likewise, the function that CAR has in mediating in vivo gene delivery by Ad vectors is being recognized to be increasingly complex. Our earlier work, using model cell lines, indicated that CAR expression is an important variable for efficient gene delivery by Ad vectors. ${ }^{20}$ However, we and others have also previously reported that Ad interactions with high-abundance plasma proteins (albumin and globulins) or stromal components possibly impact the bioavailability of the Ad vector in situ. ${ }^{33}$ Adding to this complexity is a recent report that suggests that CAR expression on erythrocytes possibly serves as a sink to diminish systemic delivery of the vector to target sites (eg, specific organ or tumor). ${ }^{53}$ Finally, even if bioavailability challenges can be overcome by altering the viral capsid or the host environment, in light of recognized tumor heterogeneity in lung cancer, we speculate that only a subset of cells within any tumor are likely expressing sufficient CAR and other co-receptors to mediate efficient Ad entry. Thus, even under optimal circumstances, we speculate that only a fraction of tumor cells will be targeted by any Ad or retargeted Ad approaches; however, it remains to be determined whether the fraction of cells that are transduced in this manner will yield a therapeutic benefit.

In this report, we functionally validate an earlier observation $^{34}$ that blocking CAR expression in lung cancer cells with high expression of this molecule inhibits their ability to form tumor xenografts in mice (Figures 1,2, and 3). Thus, our data from lung cancer cells suggest a role for CAR in tumor engraftment, though the mechanism underlying this role remains elusive. This is in part due to the experimental realization that assays that are typically used for ascertaining biological functions (eg, aggregation or barrier function) yield inconclusive results when lung cancer cells are used (Figure 4), and frustrate the effort to definitively ascertain CAR biology in these cells. However, if the mesenchymal cell phenotype is an accurate measure of an undifferentiated and invasive state, then CAR expression seems to be more closely aligned with this phenotype of lung cancer cells (Figure 5). Thus, if these results are an accurate reflection of CAR's biological role, then they implicate a putative role for CAR during EMT, and lend credibility to dynamically testing CAR expression in transitioning models 
of epithelia as a better indicator of CAR biology in tumor tissues.

That an cell adhesion molecule like CAR would participate in tumor progression is not surprising, and there is ample precedence for the aberrant expression or activity of adhesion molecules associated with tumor progression. ${ }^{54}$ For example, occludin and other $\mathrm{TJ}$ components are displaced in rastransformed MDCK cells, ${ }^{55}$ and claudin 1 proteins are frequently dysregulated and overexpressed with tumor progression. ${ }^{56-60}$ Among the single-pass epithelial transmembrane TJ proteins, JAM, which recruits occludin, claudin, ZO1, and interacts with the PAR complex for junctional assembly and epithelial polarity, is the closest structural analogue of CAR. ${ }^{61-63}$ Like CAR, JAM is also suggested to be involved in the EMT during embryonic development. ${ }^{64}$ In adult tissues, JAM participates in epithelial-endothelial and epithelial-stromal cell interactions, ${ }^{65}$ which can lend themselves to participate in pathological processes, including tumor progression. Thus, JAM-C expression may mediate metastatic spread, ${ }^{66}$ and antibody neutralization of JAM-C reduces tumor growth, decreases macrophages infiltration, and inhibits angiogenesis. ${ }^{67}$ Interestingly, recent studies suggest that JAM isoforms also interact with CAR in a heterophilic manner to mediate heterotypic cell interactions. ${ }^{10,68}$

Thus, accruing data seem to suggest a more promiscuous function for CAR in mediating interactions between distinct cellular species and tissue origins. Based on these data, we suggest that the characterization of CAR as a homotypic adhesion molecule needs to be broadened. ${ }^{3,4}$ How this role is subverted during malignant progression is unknown, but to associate CAR with the 'differentiated state' (because it is an epithelial adhesion molecule in polarized epithelia) is likely incomplete, or incorrect. To suggest that CAR functions solely as a mediator of barrier function in polarized epithelia also does not appear to be consistent with its emerging portrait as a developmentally expressed adhesion molecule in the mammalian brain, ${ }^{69-71}$ heart, ${ }^{8,72,73}$ and skeletal muscle, ${ }^{74}$ nor with its characterization as a mediator of heterotypic cell interactions. ${ }^{10,68}$ The fact that CAR is ubiquitously expressed in both epithelium- and mesenchyme-derived tissues (apparently including hematopoietic precursors ${ }^{53}$ ) during development suggests a more generalized role for CAR. In the adult organism, perhaps, the reexpression of this molecule in epithelial tissues is indicative of a recapitulation of ontogeny following epithelial injury, and/or tumor progression.

\section{ACKNOWLEDGEMENT}

This project was supported from the Veterans Administration Medical Research Funds. We thank Dr Ling Zhang for the outstanding technical support.

1. Sollerbrant $\mathrm{K}$, Raschperger $\mathrm{E}, \mathrm{Mirza} M$, et al. The coxsackievirus adenovirus receptor (CAR) forms a complex with the PDZ domaincontaining protein Ligand-of-Numb protein-X (LNX). J Biol Chem 2003;278:7439-7444.
2. Mirza $M$, Raschperger $E$, Philipson $L$, et al. The cell surface protein coxsackie-adenovirus receptor (CAR) directly associates with the Ligand-of-Numb Protein-X2 (LNX2). Exp Cell Res 2005;309:110-120.

3. Cohen CJ, Shieh JT, Pickles RJ, et al. The coxsackievirus adenovirus receptor is a transmembrane component of the tight junction. Proc Natl Acad Sci USA 2001;98:15191-15196.

4. Walters RW, Freimuth $P$, Moninger TO, et al. Adenovirus fiber disrupts CAR-mediated intercellular adhesion allowing virus escape. Cell 2002;110:789-799.

5. Bergelson JM, Cunningham JA, Droguett $G$, et al. Isolation of a common receptor for coxsackie $B$ viruses adenoviruses 2 and 5 . Science 1997;275:1320-1323.

6. Dorner AA, Wegmann F, Butz S, et al. Coxsackievirus-adenovirus receptor (CAR) is essential for early embryonic cardiac development. J Cell Sci 2005;118:3509-3521.

7. Asher DR, Cerny AM, Weiler SR, et al. Coxsackievirus adenovirus receptor is essential for cardiomyocyte development. Genesis 2005;42:77-85.

8. Chen JW, Zhou B, Yu QC, et al. Cardiomyocyte-specific deletion of the coxsackievirus adenovirus receptor results in hyperplasia of the embryonic left ventricle abnormalities of sinuatrial valves. Circ Res 2006;98:923-930.

9. Philipson L, Pettersson RF. The coxsackie-adenovirus receptor-a new receptor in the immunoglobulin family involved in cell adhesion. Curr Top Microbiol Immunol 2004;273:87-111.

10. Zen K, Liu Y, McCall IC, et al. Neutrophil migration across tight junctions is mediated by adhesive interactions between epithelial coxsackie adenovirus receptor a junctional adhesion molecule-like protein on neutrophils. Mol Biol Cell 2005;16:2694-2703.

11. Excoffon KJ, Hruska-Hageman A, Klotz $M$, et al. A role for the PDZ-binding domain of the coxsackie $B$ virus adenovirus receptor (CAR) in cell adhesion and growth. J Cell Sci 2004;117: 4401-4409.

12. Cohen CJ, Gaetz J, Ohman T, et al. Multiple regions within the coxsackievirus adenovirus receptor cytoplasmic domain are required for basolateral sorting. J Biol Chem 2001;20:20.

13. Coyne $\mathrm{CB}$, Voelker T, Pichla SL, et al. The coxsackievirus adenovirus receptor interacts with the multi-PDZ domain protein-1 (MUPP-1) within the tight junction. J Biol Chem 2004;279:48079-48084.

14. Mirza M, Hreinsson J, Strand ML, et al. Coxsackievirus adenovirus receptor (CAR) is expressed in male germ cells and forms a complex with the differentiation factor JAM-C in mouse testis. Exp Cell Res 2006;312:817-830.

15. Fok PT, Huang KC, Holland PC, et al. The coxsackie adenovirus receptor (CAR) binds microtubules and plays a role in cell migration. J Biol Chem 2007;282:7512-7521.

16. Okegawa T, Li Y, Pong RC, et al. The dual impact of coxsackie adenovirus receptor expression on human prostate cancer gene therapy. Cancer Res 2000;60:5031-5036.

17. Okegawa T, Pong RC, Li Y, et al. The mechanism of the growthinhibitory effect of coxsackie adenovirus receptor (CAR) on human bladder cancer: a functional analysis of car protein structure. Cancer Res 2001;61:6592-6600.

18. Li Y, Pong RC, Bergelson JM, et al. Loss of adenoviral receptor expression in human bladder cancer cells: a potential impact on the efficacy of gene therapy. Cancer Res 1999;59:325-330.

19. Batra RK, Olsen JC, Pickles RJ, et al. Transduction of non-small cell lung cancer cells by adenoviral retroviral vectors. Am J Respir Cell Mol Biol 1998;18:402-410.

20. Qin M, Chen S, Yu T, et al. Coxsackievirus adenovirus receptor expression predicts the efficiency of adenoviral gene transfer into nonsmall cell lung cancer xenografts. Clin Cancer Res 2003;9:4992-4999.

21. Gu W, Ogose A, Kawashima $\mathrm{H}$, et al. High-level expression of the coxsackievirus adenovirus receptor messenger RNA in osteosarcoma Ewing's sarcoma, and benign neurogenic tumors among musculoskeletal tumors. Clin Cancer Res 2004;10:3831-3838.

22. Rauen KA, Sudilovsky D, Le JL, et al. Expression of the coxsackie adenovirus receptor in normal prostate in primary metastatic prostate carcinoma: potential relevance to gene therapy. Cancer Res 2002;62:3812-3818

23. Fuxe J, Liu L, Malin S, et al. Expression of the coxsackie adenovirus receptor in human astrocytic tumors xenografts. Int J Cancer 2003;103:723-729. 
24. Hemmi S, Geertsen R, Mezzacasa A, et al. The presence of human coxsackievirus adenovirus receptor is associated with efficient adenovirus-mediated transgene expression in human melanoma cell cultures. Hum Gene Ther 1998;9:2363-2373.

25. Miller CR, Buchsbaum DJ, Reynolds PN, et al. Differential susceptibility of primary established human glioma cells to adenovirus infection: targeting via the epidermal growth factor receptor achieves fiber receptor-independent gene transfer. Cancer Res 1998:58:5738-5748.

26. Cripe TP, Dunphy EJ, Holub AD, et al. Fiber knob modifications overcome low heterogeneous expression of the coxsackievirusadenovirus receptor that limits adenovirus gene transfer oncolysis for human rhabdomyosarcoma cells. Cancer Res 2001;61:2953-2960.

27. Douglas JT, Kim M, Sumerel LA, et al. Efficient oncolysis by a replicating adenovirus (ad) in vivo is critically dependent on tumor expression of primary ad receptors. Cancer Res 2001;61:813-817.

28. Anders M, Hansen R, Ding RX, et al. Disruption of 3D tissue integrity facilitates adenovirus infection by deregulating the coxsackievirus adenovirus receptor. Proc Natl Acad Sci USA 2003;100:1943-1948.

29. Lacher MD, Tiirikainen MI, Saunier EF, et al. Transforming growth factor-beta receptor inhibition enhances adenoviral infectability of carcinoma cells via up-regulation of coxsackie adenovirus receptor in conjunction with reversal of epithelial-mesenchymal transition. Cancer Res 2006;66:1648-1657.

30. Bruning A, Stickeler E, Diederich D, et al. Coxsackie adenovirus receptor promotes adenocarcinoma cell survival is expressionally activated after transition from preneoplastic precursor lesions to invasive adenocarcinomas. Clin Cancer Res 2005;11:4316-4320.

31. Korn WM, Macal M, Christian C, et al. Expression of the coxsackievirusadenovirus receptor in gastrointestinal cancer correlates with tumor differentiation. Cancer Gene Ther 2006.

32. Sachs MD, Rauen KA, Ramamurthy $M$, et al. Integrin alpha(v) and coxsackie adenovirus receptor expression in clinical bladder cancer. Urology 2002;60:531-536.

33. Bernal RM, Sharma S, Gardner BK, et al. Soluble coxsackievirus adenovirus receptor is a putative inhibitor of adenoviral gene transfer in the tumor milieu. Clin Cancer Res 2002;8:1915-1923.

34. Qin $M$, Escuadro B, Dohadwala $M$, et al. A novel role for coxsackie adenovirus receptor in mediating tumor formation by lung cancer cells. Cancer Res 2004;64:6377-6380.

35. Liu L, Mul FP, Lutter R, et al. Transmigration of human neutrophils across airway epithelial cell monolayers is preferentially in the physiologic basolateral-to-apical direction. Am J Respir Cell Mol Biol 1996;15:771-780.

36. Winton $\mathrm{HL}$, Wan $\mathrm{H}$, Cannell MB, et al. Cell lines of pulmonary nonpulmonary origin as tools to study the effects of house dust mite proteinases on the regulation of epithelial permeability. Clin Exp Allergy 1998;28:1273-1285.

37. Molock KE, Lillehoj EP. Biochemical interactions among intercellular adhesion molecules expressed by airway epithelial cells. Biochem Biophys Res Commun 2006;343:513-519.

38. Kim S, Schein AJ, Nadel JA. E-cadherin promotes EGFR-mediated cell differentiation MUC5AC mucin expression in cultured human airway epithelial cells. Am J Physiol Lung Cell Mol Physiol 2005;289: L1049-L1060.

39. Grainger $\mathrm{Cl}$, Greenwell LL, Lockley DJ, et al. Culture of Calu-3 cells at the air interface provides a representative model of the airway epithelial barrier. Pharm Res 2006;23:1482-1490.

40. Reddy MM, Quinton PM, Haws C, et al. Failure of the cystic fibrosis transmembrane conductance regulator to conduct ATP. Science 1996;271:1876-1879.

41. Hsu KH, Lonberg-Holm K, Alstein B, et al. A monoclonal antibody specific for the cellular receptor for the group $B$ coxsackieviruses. J Virol 1988;62:1647-1652.

42. Rozanov DV, Deryugina El, Monosov EZ, et al. Aberrant persistent inclusion into lipid rafts limits the tumorigenic function of membrane type-1 matrix metalloproteinase in malignant cells. Exp Cell Res 2004:293:81-95.

43. Dohadwala Mariam S-CY, Luo J, Sharma S, et al. Cyclooxygenase-2dependent regulation of E-cadherin: prostaglandin E2 induces transcriptional repressors ZEB1 Snail in non-small cell lung cancer. Cancer Res 2006;66:5338-5345.
44. Nightingale J, Chaudhary KS, Abel PD, et al. Ligand activation of the androgen receptor downregulates E-cadherin-mediated cell adhesion promotes apoptosis of prostatic cancer cells. Neoplasia 2003;5: 347-361.

45. Qin M, Escuadro B, Sharma $S$, et al. Gene transfer mediated by native versus fibroblast growth factor-retargeted adenoviral vectors into lung cancer cells. Am J Respir Cell Mol Biol 2005;32:211-217.

46. van Raaij MJ, Chouin E, van der Zandt $H$, et al. Dimeric structure of the coxsackievirus adenovirus receptor D1 domain at 17 A resolution. Struct Fold Des 2000;8:1147-1155.

47. Lortat-Jacob H, Chouin E, Cusack S, et al. Kinetic analysis of adenovirus fiber binding to its receptor reveals an avidity mechanism for trimeric receptor-ligand interactions. J Biol Chem 2001;276:9009-9015.

48. Bren-Mattison $\mathrm{Y}$, Van Putten V, Chan D, et al. Peroxisome proliferator-activated receptor-gamma (PPAR(gamma)) inhibits tumorigenesis by reversing the undifferentiated phenotype of metastatic non-small-cell lung cancer cells (NSCLC). Oncogene 2005;24:1412-1422.

49. Thomson S, Buck E, Petti F, et al. Epithelial to mesenchymal transition is a determinant of sensitivity of non-small-cell lung carcinoma cell lines xenografts to epidermal growth factor receptor inhibition. Cancer Res 2005;65:9455-9462.

50. Martin TA, Watkins G, Jiang WG. The coxsackie-adenovirus receptor has elevated expression in human breast cancer. Clin Exp Med 2005;5:122-128.

51. Wang $\mathrm{Y}$, Wang $\mathrm{S}, \mathrm{Bao} \mathrm{Y}$, et al. Coxsackievirus adenovirus receptor expression in non-malignant lung tissues clinical lung cancers. J Mol Histol 2006;37:153-160.

52. Giaginis CT, Zarros AC, Papaefthymiou MA, et al. Coxsackievirus adenovirus receptor expression in human endometrial adenocarcinoma: possible clinical implications. World J Surg Oncol 2008;6:59.

53. Seiradake $E$, Henaff $D$, Wodrich $H$, et al. The cell adhesion molecule 'CAR' and sialic acid on human erythrocytes influence adenovirus in vivo biodistribution. PLoS Pathog 2009;5:e1000277.

54. Tsukita S, Yamazaki Y, Katsuno T, et al. Tight junction-based epithelial microenvironment cell proliferation. Oncogene 2008;27:6930-6938.

55. Chen $\mathrm{Y}, \mathrm{Lu} \mathrm{Q}, \mathrm{Schneeberger} \mathrm{EE}$, et al. Restoration of tight junction structure barrier function by down-regulation of the mitogenactivated protein kinase pathway in ras-transformed Madin-Darby canine kidney cells. Mol Biol Cell 2000;11:849-862.

56. Johnson $A H$, Frierson HF, Zaika $A$, et al. Expression of tight-junction protein claudin-7 is an early event in gastric tumorigenesis. Am J Pathol 2005;167:577-584.

57. Lee JW, Lee SJ, Seo J, et al. Increased expressions of claudin-1 claudin-7 during the progression of cervical neoplasia. Gynecol Oncol 2005;97:53-59.

58. Sobel G, Paska C, Szabo I, et al. Increased expression of claudins in cervical squamous intraepithelial neoplasia invasive carcinoma. Hum Pathol 2005;36:162-169.

59. Oku N, Sasabe E, Ueta E, et al. Tight junction protein claudin-1 enhances the invasive activity of oral squamous cell carcinoma cells by promoting cleavage of laminin-5 gamma2 chain via matrix metalloproteinase (MMP)-2 and membrane-type MMP-1. Cancer Res 2006;66:5251-5257.

60. Dhawan $P$, Singh $A B$, Deane NG, et al. Claudin-1 regulates cellular transformation metastatic behavior in colon cancer. J Clin Invest 2005;115:1765-1776.

61. Liu Y, Nusrat A, Schnell FJ, et al. Human junction adhesion molecule regulates tight junction resealing in epithelia. J Cell Sci 2000;113:2363-2374.

62. Ebnet K, Suzuki A, Horikoshi Y, et al. The cell polarity protein ASIP/PAR3 directly associates with junctional adhesion molecule (JAM). EMBO J 2001;20:3738-3748.

63. Ebnet $K$, Aurrand-Lions $M$, Kuhn $A$, et al. The junctional adhesion molecule (JAM) family members JAM-2 and JAM-3 associate with the cell polarity protein PAR-3: a possible role for JAMs in endothelial cell polarity. J Cell Sci 2003;116:3879-3891.

64. Thomas FC, Sheth B, Eckert JJ, et al. Contribution of JAM-1 to epithelial differentiation tight-junction biogenesis in the mouse preimplantation embryo. J Cell Sci 2004;117:5599-5608. 
65. Morris AP, Tawil A, Berkova $\mathrm{Z}$, et al. Junctional adhesion molecules (JAMs) are differentially expressed in fibroblasts and co-localize with ZO-1 to adherens-like junctions. Cell Commun Adhes 2006; 13:233-247.

66. Santoso S, Orlova VV, Song K, et al. The homophilic binding of junctional adhesion molecule- $\mathrm{C}$ mediates tumor cell-endothelial cell interactions. J Biol Chem 2005;280:36326-36333.

67. Lamagna C, Hodivala-Dilke KM, Imhof BA, et al. Antibody against junctional adhesion molecule- $C$ inhibits angiogenesis tumor growth. Cancer Res 2005;65:5703-5710.

68. Luissint AC, Lutz PG, Calderwood DA, et al. JAM-L-mediated leukocyte adhesion to endothelial cells is regulated in cis by alpha4beta1 integrin activation. J Cell Biol 2008;183:1159-1173.

69. Xu R, Crowell RL. Expression distribution of the receptors for coxsackievirus B3 during fetal development of the Balb/c mouse of their brain cells in culture. Virus Res 1996;46: 157-170.
70. Honda $\mathrm{T}$, Saitoh $\mathrm{H}$, Masuko $\mathrm{M}$, et al. The coxsackievirus-adenovirus receptor protein as a cell adhesion molecule in the developing mouse brain. Brain Res Mol Brain Res 2000;77:19-28.

71. Hotta Y, Honda T, Naito M, et al. Developmental distribution of coxsackie virus adenovirus receptor localized in the nervous system. Brain Res Dev Brain Res 2003;143:1-13.

72. Ito $M$, Kodama $M$, Masuko $M$, et al. Expression of coxsackievirus adenovirus receptor in hearts of rats with experimental autoimmune myocarditis. Circ Res 2000;86:275-280.

73. Kashimura $T$, Kodama M, Hotta $Y$, et al. Spatiotemporal changes of coxsackievirus adenovirus receptor in rat hearts during postnatal development in cultured cardiomyocytes of neonatal rat. Virchows Arch 2004;444:283-292.

74. Nalbantoglu J, Pari G, Karpati G, et al. Expression of the primary coxsackie adenovirus receptor is downregulated during skeletal muscle maturation limits the efficacy of adenovirus-mediated gene delivery to muscle cells. Hum Gene Ther 1999;10:1009-1019. 